\title{
Modeling neuronal dynamics during brain ischemia
}

\author{
Bas-Jan Zandt ${ }^{1,2}$, Bennie ten Haken ${ }^{1,2}$, Michel JAM van Putten ${ }^{2,3}$ \\ From Twentieth Annual Computational Neuroscience Meeting: CNS*2011 \\ Stockholm, Sweden. 23-28 July 2011
}

Neurons are critically dependent on sufficient supply of oxygen and glucose When this supply is subcritical, as in ischemia, the signaling processes of the neurons are altered due to synaptic transmission failure [1] and decreased rates of the molecular ion pumps needed to maintain transmembrane ion gradients [2]. These changes are partially reflected in the electroencephalogram (EEG), as this is a sensitive measure for global network function, reflecting extracellular currents, mainly from synchronous activity of pyramidal cells [3]. Modeling a neural network suffering from ischemia may further improve our understanding of the effects of ischemia and the relation with the EEG. This may contribute to improved methods for diagnosing pathologies related to ischemia and hypoxia.

The electrophysiological behavior of a single neuron, which we calculate with a Hodgkin-Huxley type model, depends heavily on the intra- and extracellular ion concentrations. These are usually assumed to be constant, because the molecular ion pumps (ATPases) of the neurons and glia maintain the concentration differences needed for the generation of action potentials. During ischemia however, the required energy (ATP) for the pumping process is not fully supplied anymore and these concentrations change. This is calculated by integrating the average fluxes of the individual ion species across the cell membranes, following an approach similar to those used by Dronne [4] and Cressman [5].

The pump rates are assumed to be linear with the total energy use of a cell, for which Michaelis-Menten kinetics depending on the oxygen concentration are assumed. These concentrations depend on the position of the cell relative to the blood vessels.
With a model for the behavior of a single neuron during hypoxia, the behavior of a network of these neurons, perhaps with different properties, and the resulting EEG signals can be simulated.

We will present our first results of the influence on the electrical activity of the oxygen supply to the cells in the network.

\section{Author details}

${ }^{1}$ Department of Applied Physics, University of Twente, Enschede, The Netherlands. ${ }^{2}$ MIRA-Institute for Biomedical Technology and Technical Medicine, University of Twente, Enschede, The Netherlands. ${ }^{3}$ Department of Clinical Neurophysiology, Medisch Spectrum Twente, Enschede, The Netherlands.

Published: 18 July 2011

References

1. Bolay H, Gürsoy-Ozdemir Y, Sara Y, Onur R, Can A, Dalkara T: Persistent Defect in Transmitter Release and Synapsin Phosphorylation in Cerebral Cortex After Transient Moderate Ischemic Injury. Stroke 2002, 33:1369-1375.

2. Krnjevic K: Early Effects of Hypoxia on Brain Cell Function. Croat Med J 1999, 40(3):375-380.

3. Visser S, Meijer HG, Lee HC, van Drongelen W, van Putten MJ, van Gils SA: Comparing epileptiform behavior of mesoscale detailed models and population models of neocortex. J Clin Neurophysio 2010, 27(6):471-8.

4. Dronne MA, Boissel JP, Grenier E: A mathematical model of ion movements in grey matter during a stroke. J Theor Biol 2006, 240(4):599-615.

5. Cressman JR, Ullah G, Ziburkus J, Schiff SJ, Barreto E: The influence of sodium and potassium dynamics on excitability, seizures, and the stability of persistent states: I. Single neuron dynamics. J Comput Neurosci 2009, 26:159-170.

\section{doi:10.1186/1471-2202-12-S1-P354}

Cite this article as: Zandt et al.: Modeling neuronal dynamics during brain ischemia. BMC Neuroscience 2011 12(Suppl 1):P354.

\footnotetext{
* Correspondence: b.zandt@utwente.nl

'Department of Applied Physics, University of Twente, Enschede, The Netherlands

Full list of author information is available at the end of the article
} 